\title{
Effect of the burnable absorber arrangement on the VVER-1200 fuel assembly neutronic performance*
}

\author{
Ruslan A. Vnukov ${ }^{1}$, Valery V. Kolesov², Irina A. Zhavoronkova ${ }^{1}$, \\ Yaroslav A. Kotov ${ }^{3}$, Md Masum Rana Pramanik ${ }^{2}$ \\ 1 Obninsk Institute for Nuclear Power Engineering NRNU MEPhI, 1 Studgorodok, 249040 Obninsk, Kaluga Reg., Russia \\ 2 National Research Nuclear University MEPhI, 31 Kashirskoe Hwy, 115409 Moscow, Russia \\ 3 NRC Kurchatov Institute, 1 Academician Kurchatov Sq., 123182 Moscow, Russia \\ Corresponding author: Ruslan A. Vnukov (levz555@mail.ru)
}

Academic editor: Georgy Tikhomirov • Received 11 November 2020 Accepted 13 July 2021 • Published 23 September 2021

Citation: Vnukov RA, Kolesov VV, Zhavoronkova IA, Kotov YaA, Pramanik Md MR (2021) Effect of the burnable absorber arrangement on the VVER-1200 fuel assembly neutronic performance. Nuclear Energy and Technology 7(3): 215-221. https://doi. org/10.3897/nucet.7.73490

\begin{abstract}
Optimizing the use of fuel in a power reactor is a task of current concern. However, little attention has been given to investigating the dependences among the enrichment used, the content of gadolinium oxide in fuel elements, and the life time in combination with assessing the efficiency of using $\mathrm{Gd}$ fuel elements with different $\mathrm{Gd}_{2} \mathrm{O}_{3}$ contents.

The paper considers fuel assembly (FA) versions for VVER-1200 reactors having different enrichments for fuel elements, including those with $\mathrm{Gd}$, and different contents of gadolinium oxide in fuel. A comparative analysis is presented for assemblies with homogeneous $\mathrm{Gd}_{2} \mathrm{O}_{3}$ arrangements in each fuel element and with profiled $\mathrm{Gd}_{2} \mathrm{O}_{3}$ arrangements. In the latter case, profiling depends on the neutron flux density in the layer which includes Gd fuel elements. This suggests that the arrangement of gadolinium oxide proportionally to the neutron flux density will improve the FA neutronic performance.

The results were obtained using SERPENT (a continuous-energy multi-purpose three-dimensional Monte Carlo particle transport code). The assemblies with the used parameters for a 12-month fuel cycle have shown the method under consideration to be inefficient for a period of over 300 eff. days. With increased enrichment and content of gadolinium oxide, the use of profiled versions has turned out to be more rational for longer periods (up to 900 eff. days). Therefore, this phenomenon is relevant for the reactor life, whereas it proves to be insignificant for the fuel life. A complex relationship is noted between the gadolinium and uranium content in an assembly and the effective multiplication factor for the profiled and standard assemblies. This relationship requires further detailed consideration.
\end{abstract}

\section{Keywords}

Burnable absorber, optimal profiling, fuel assembly, neutron flux density, Monte Carlo method

\section{Introduction}

Burnable absorbers are used in view of the need to compensate for excess reactivity due to safety reasons during the operation of a nuclear power plant. This can be interpreted as an alternative to the use of boron control (regarding pressurized water reactors) as well as to the extension of fuel or reactor campaigns. The

* Russian text published: Izvestiya vuzov. Yadernaya Energetika (ISSN 0204-3327), 2021, n. 2, pp. 27-38.

Copyright Vnukov RA et al. This is an open access article distributed under the terms of the Creative Commons Attribution License (CC-BY 4.0), which permits unrestricted use, distribution, and reproduction in any medium, provided the original author and source are credited. 


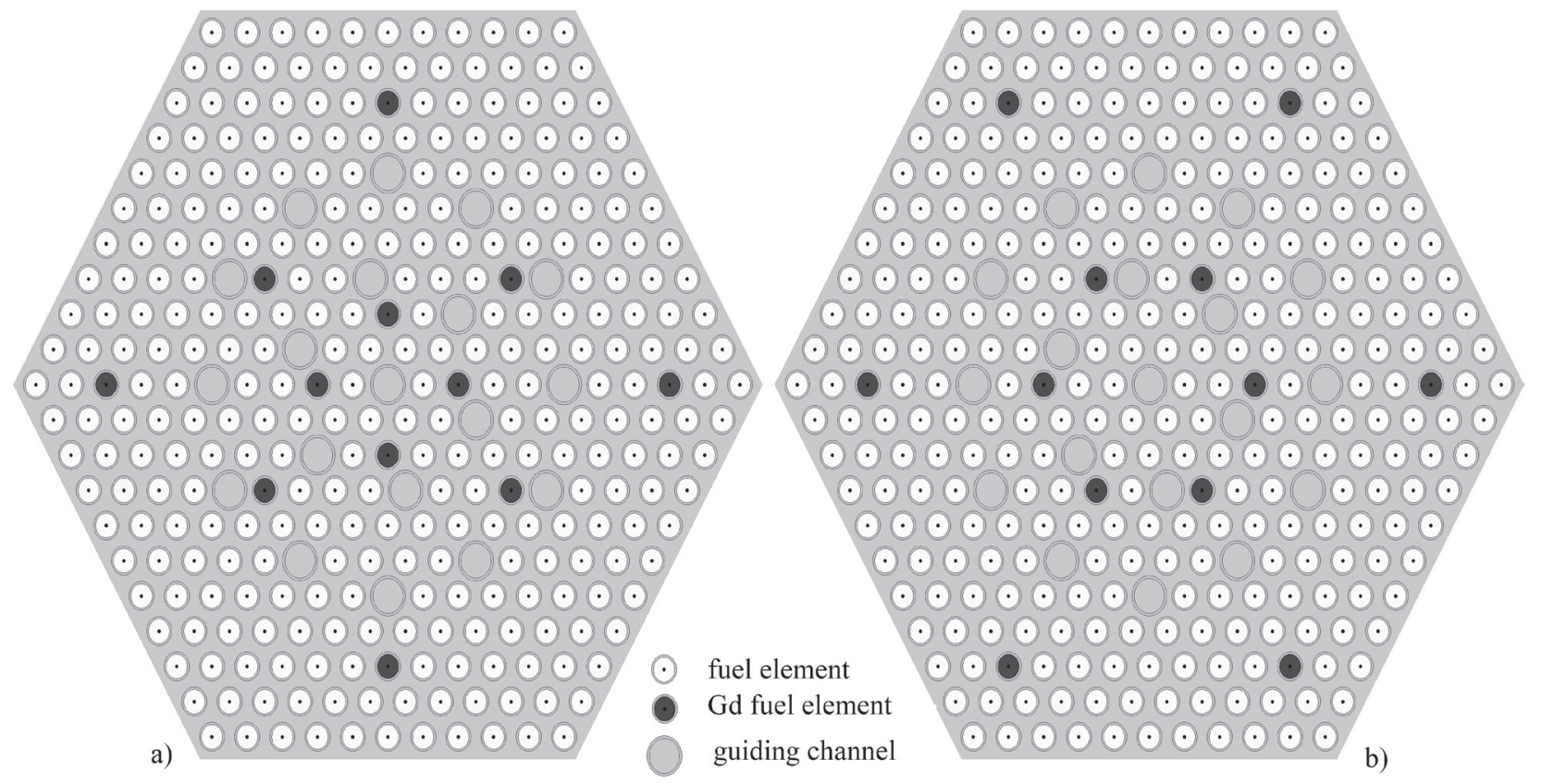

Figure 1. Gd fuel element arrangement options: a) in the third, sixth and ninth rings of the fuel assembly; b) in the third and eighth rings (counting from the periphery, top view).

influence on the campaign parameters was considered in (Khoshahval et al. 2016, Abu Sondos et al. 2019a, 2019b, 2019c, Frybortova 2019, Saad et al. 2019). Research is also conducted in terms of assessing the behavior of uranium-gadolinium fuel during homogeneous mixing of elements: when gadolinium is added to the fuel, its thermal conductivity changes, affecting the design characteristics. At a $\mathrm{Gd}_{2} \mathrm{O}_{3}$ concentration of about $10 \%$ and a pellet density of $10.62 \mathrm{~g} / \mathrm{cm}^{3}$, high thermal conductivity is maintained (Iwasaki et al. 2009). Further changes in these values lead to a decrease in this parameter value. The ratio of the uranium and gadolinium fractions in fuel is relevant from the standpoint of its burnup in reactors.

The $\mathrm{Gd}_{2} \mathrm{O}_{3}$ content reaches 9 wt. $\%$ in standard fuel elements (Slivin et al. 2016) and 12 wt.\% in experimental ones (Kryukov et al. 2005). This study represents a consideration of new fuel compositions with a burnable absorber and includes reactor calculations of neutronic processes based on the Monte Carlo method. The goal is to determine the possibility of extending the campaign by means of burnable absorbers. The fuel burns in such a way that, during operation, an increasing number of U-235 fissions occurs in the deeper layers of the fuel element ( $\mathrm{Gd}$ fuel element). However, the assembly, due to uneven burnup, is presumably removed before the moment when all the stored reactivity is released. As a result, the efficiency of using burnable absorbers is reduced.

\section{Problem setting}

If the burnup of gadolinium is considered on the scale of fuel assemblies, then there appear regularities, the use of which in profiling will, as expected, give a positive result in the matter of extending the campaign.

The paper investigates various gadolinium arrangement options in fuel assemblies. The initial assembly was a standard VVER-1200 fuel assembly with 12 Gd fuel elements arranged at the corners of the hexagon in the third and eighth rings (Fig. 1).

In each fuel element, gadolinium oxide is uniformly mixed with uranium fuel. The $\mathrm{Gd}_{2} \mathrm{O}_{3}$ content is $8 \%$. The density power was taken equal to $36 \mathrm{~kW} / \mathrm{kg}$ of fuel, which corresponds to the average performance of VVER-1200. The maximum campaign time was taken equal to 3360 eff. days. The most interesting were the intervals $0-360$ and 0-560 eff. days.

Profiling was carried out as follows: the neutron flux density was estimated at the time of initial loading in each hexagonal ring, the starting concentrations of gadolinium oxide and uranium dioxide were set in proportion to the neutron flux density, after which the output data were compared with the standard assembly when the concentrations were not changed.

The use of a burnable absorber makes it possible to reduce the amount of boric acid in the coolant or, in general, to increase the reactivity margin when using the same boric acid concentration and higher fuel enrichment. After the data on the burnup of the initial assembly were obtained, the task was to find the most optimal placement. After the data were obtained with fuel of a given enrichment, the concentration of uranium-235 changed, both in the fuel elements and in the gadolinium fuel elements. This was done to confirm the following theses:

1. an increase in fuel enrichment (in fuel elements and Gd fuel elements) increases the time when profiling of 
gadolinium by neutron flux density is a more efficient option in cases where microcampaigns for more than one year (more than 360 eff. days) are considered;

2. an increase in the number of hexagonal rings where Gd fuel elements are arranged, with their total number remaining unchanged, is optimal both for profiled fuel assemblies and assemblies with the same concentrations; and

3. an increase in the concentration of gadolinium in fuel assemblies for profiling (with respect to the uniform distribution of $\mathrm{Gd}_{2} \mathrm{O}_{3}$ in each $\mathrm{Gd}$ fuel element) gives a positive effect (higher $k_{\text {eff }}$ ) by the end of the microcampaign for a longer time.

The last thesis requires a search for the maximum concentration of gadolinium oxide at which the profiling effect on a prolonged campaign will be positive. This is due to the fact that as the amount of gadolinium in the Gd fuel element increases, the total amount of uranium decreases. And since a decrease in the concentration of uranium-235 reduces the profiling efficiency, this becomes an optimization problem.

For this reason, we shall consider the assemblies presented in Table 1.

Table 1. Options of simulated fuel assemblies

\begin{tabular}{lccc}
\hline $\begin{array}{c}\text { Number of rings } \\
\text { (Nos.) }\end{array}$ & $\begin{array}{c}\text { Gadolinium oxide } \\
\text { content, } \%\end{array}$ & $\begin{array}{c}\text { Fuel element } \\
\text { enrichment, } \%\end{array}$ & $\begin{array}{c}\text { Gd fuel element } \\
\text { enrichment, \% }\end{array}$ \\
\hline $2(3,8)$ & 8 & 4 & 4 \\
$2(3,8)$ & 8 & 4.95 & 4 \\
$2(3,8)$ & 8 & 4.95 & 4.95 \\
$2(3,8)$ & 8 & 4.95 & 0.25 \\
$2(4,9)$ & 8 & 4.95 & 4 \\
$3(3,6,9)$ & 8 & 4.95 & 4.95 \\
$2(3,8)$ & 12 & 4 & 4 \\
$3(3,6,9)$ & 12 & 4 & 4 \\
$3(3,6,9)$ & 13.5 & 4.95 & 4.95 \\
$2(3,8)$ & 13.5 & 4.95 & 5.2 \\
$3(3,6,9)$ & 13.5 & 4.95 & 5.2 \\
$2(3,8)$ & 13.5 & 5.2 & 5.2 \\
$3(3,6,9)$ & 13.5 & 5.2 & 5.2 \\
$2(3,8)$ & 13.5 & 5.45 & 5.45 \\
$3(3,6,9)$ & 13.5 & 5.45 & 5.45 \\
$2(3,8)$ & 16 & 5.45 & 5.45 \\
$3(3,6,9)$ & 16 & 5.45 & 5.45 \\
$2(3,8)$ & 20 & 5.45 & 5.45 \\
$3(3,6,9)$ & 20 & 5.45 & 5.45 \\
$2(3,8)$ & 24 & 5.45 & 5.45 \\
$3(3,6,9)$ & 24 & 5.45 & 5.45 \\
\hline & & &
\end{tabular}

In terms of the number of rings, given the number of Gd fuel elements (12 pcs), two options turned out to be acceptable: two and three rings with Gd fuel elements (four elements in a hexagonal ring).

The enrichment of fuel elements and Gd fuel elements was considered separately, since two aspects were assessed: (1) efficiency of a profiled assembly with higher enrichment of fuel elements and (2) efficiency of Gd fuel elements with an additional reactivity margin.

The work was carried out in the computing environment of SERPENT (a continuous-energy multi-purpose three-dimensional Monte Carlo particle transport code) (SERPENT - MCRPBCC 2020).

\section{Results and discussion}

Let us now define an alphanumeric string format that will make it easier for the reader to perceive the characteristics of assemblies in the graphs in this section:

NR_AV_tvel_EN tvel-tveg_EN tveg_ OP[_AN][ TRP][AR].

The meaning of the chain items in bold is as follows:

- NR is the number of rings containing Gd fuel elements, and the numbering of these rings. For example, " $83 "=\mathrm{Gd}$ fuel elements are arranged in Rings 3 and 8, counting from the FA periphery; " $369 "=\mathrm{Gd}$ fuel elements are placed in Rings 3, 6 and 9.

- AV is the assembly version: "st" denotes a standard assembly, where the gadolinium oxide content in each Gd fuel element is the same, and "pr" is a profiled assembly, where the concentrations in Gd fuel elements differ in proportion to the neutron flux density.

- $\mathbf{E N}_{\text {tvel }}$ is percentage enrichment of fuel elements (for example, $4.95 \%$ is written as 4-95).

- $\mathbf{E N}_{\text {tveg }}$ is percentage enrichment of gadolinium fuel elements (for example, 5.45\% is written as 5-45).

- $\mathbf{O P}$ is the gadolinium oxide percentage in a Gd fuel element.

- AN is the assembly numbering.

- TRP is the time-reference point ( 0 eff. days, 360, $560, \ldots)$. It is indicated when changes in the neutron flux density are considered (for example, 560 eff. days is written as 560d).

- $\mathbf{A R}$ is the gadolinium fuel element arrangement in the fuel assemble (inner, middle or outer hexagonal ring). It is indicated when the isotopic composition of Gd-155 is considered.

The items in square brackets are optional.

For example: A chain of the form 38_st_tvel_4_ tveg_4_8 means that the gadolinium fuel elements are arranged in Ring 3 and 8 from the periphery in a standard assembly, the fuel element enrichment is $4 \%$, the gadolinium fuel element enrichment is $4 \%$, and the $\operatorname{Gd}_{2} \mathrm{O}_{3}$ content is $8 \%$.

In this work, $k_{\text {eff, }}$ the neutron flux density and the calculated isotopic composition were estimated by the method proposed in (Leppänen et al. 2015). An estimate of changes in the effective multiplication factor of the first version of assemblies is shown in Fig. 2. The time interval at which $k_{\text {eff }}$ of the profiled assembly is greater than $k_{\text {eff }}$ of the standard assembly for all the options is presented in Table 2 .

It is obvious that, with its own spectrum, the profiling of Version 1 is ineffective, since during microcampaigns for more than 300 days, the standard arrangement of gadolini- 


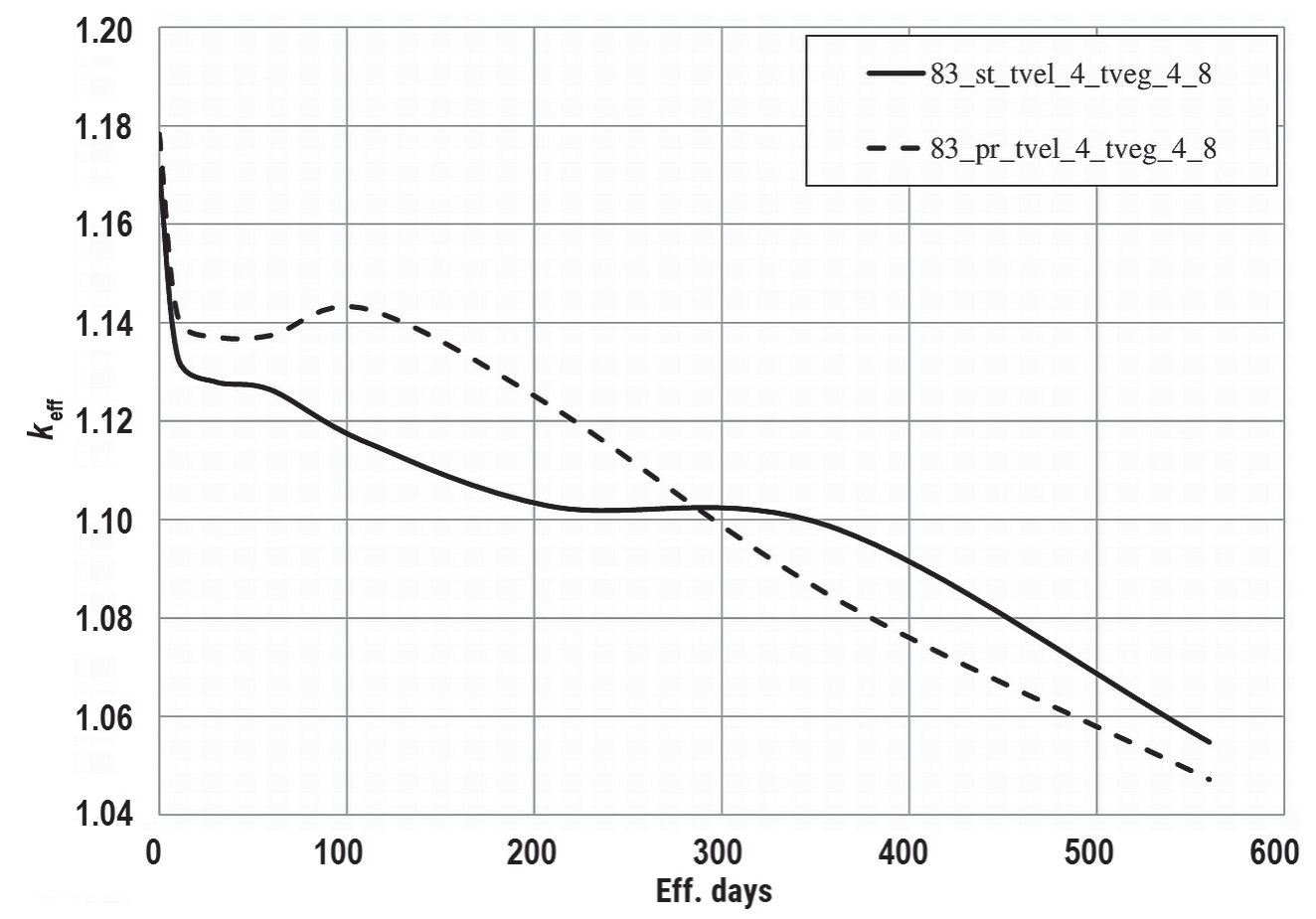

Figure 2. Changes of $k_{\text {eff }}$ for Version 1 of assemblies.

Table 2. Efficiency of profiling different versions of simulated fuel assemblies

\begin{tabular}{lcc}
\hline $\begin{array}{c}\text { Version No. }\left(\mathbf{G d}_{2} \mathbf{O}_{3}\right. \\
\text { content, \%, } \mathbf{~ F E} \\
\text { enrichment, \%, Gd }\end{array}$ & $\begin{array}{c}\text { The number of hexagonal } \\
\text { rings for the arrangement of } \\
\text { FE enrichment, \%) }\end{array}$ & $\begin{array}{c}\text { Eff. days at the } \\
\text { moment } \boldsymbol{k}_{\text {eff }} \text { (profiled) } \\
\boldsymbol{k}_{\text {eff }} \text { (standard) }\end{array}$ \\
\hline $1(8,4,4)$ & $2(3,8)$ & 291 \\
$2(8,4.95,4)$ & $2(3,8)$ & 248 \\
& $2(4,9)$ & 252 \\
$3(8,4.95,4.95)$ & $2(3,8)$ & 359 \\
$4(8,4.95,0.25)$ & $3(3,6,9)$ & 380 \\
$5(13.5,4.95,4.95)$ & $2(3,8)$ & 171 \\
$6(13.5,4.95,5.2)$ & $2(4,9)$ & 360 \\
$7(13.5,5.2,5.2)$ & $3(3,6,9)$ & 563 \\
& $2(3,8)$ & 541 \\
$8(13.5,5.45,5.45)$ & $3(3,6,9)$ & 572 \\
& $2(3,8)$ & 548 \\
$9(16,5.45,5.45)$ & $3(3,6,9)$ & 583 \\
$10(20,5.45,5.45)$ & $2(3,8)$ & 615 \\
$11(24,5.45,5.45)$ & $3(3,6,9)$ & 673 \\
$12(12,4,4)$ & $2(3,8)$ & 652 \\
& $3(3,6,9)$ & 682 \\
& $2(3,8)$ & 759 \\
& $3(3,6,9)$ & 823 \\
& $2(3,8)$ & 892 \\
& $3(3,6,9)$ & 4055 \\
& $2(3,8)$ & 462 \\
\hline
\end{tabular}

um oxide in fuel assemblies seems to be a more promising option. At the same time, data on the enrichment and content of $\mathrm{Gd}_{2} \mathrm{O}_{3}$ are relevant in VVER-1000 and VVER-1200 assemblies at the present time. Further consideration of the issue is carried out with promising assemblies that are not currently used (Stefanova et al. 1994, IAEA 2009, 2011, 2013).

For Version 2, it should be noted that there is no significant effect for profiled assemblies at a time point equal to 360 eff. days. On the other hand, within the framework of a microcampaign with higher enrichment in fuel elements, the profiled version turns out to be a more efficient option in comparison with the standard arrangement of a burnable absorber for a longer time interval.

Worth noting is that, in the case of Gd fuel elements arranged in two rings, the same situation is observed for Version 3 as in the case of their enrichment by $4 \%$. But in the case of using three rings, the intersection of the $k_{\text {eff }}$ values of the standard and profiled versions is shifted to the right side (in the region of 390 eff. days), which is a more promising option in comparison with the previous versions of assemblies.

The composition of $\mathrm{Gd}$ fuel elements of Version 4 is unpromising, since there is practically no release of reactivity. Due to the large radiative-capture crosssection, gadolinium quickly burns up, which is a negative characteristic when this absorber is used instead of, for example, a water controller or CPS. A slight plateau is observed in the first two months of operation, after which the assembly burns linearly. Plutonium production occurs in the profiled and standard assemblies in a similar way, which is reflected in the same $k_{\text {eff }}$ behavior.

In Version 5, only options with three rings are presented, since the version is intermediate, and a larger number of rings proved to be a more promising option of assemblies. In addition, we can emphasize the longer burning time of gadolinium. The intersection of $k_{\text {eff }}$ values for different assemblies goes beyond 560 eff. days, which indicates that this assembly profiling option is promising, all other things being equal, in the case of 12- and 18-month fuel cycles. 


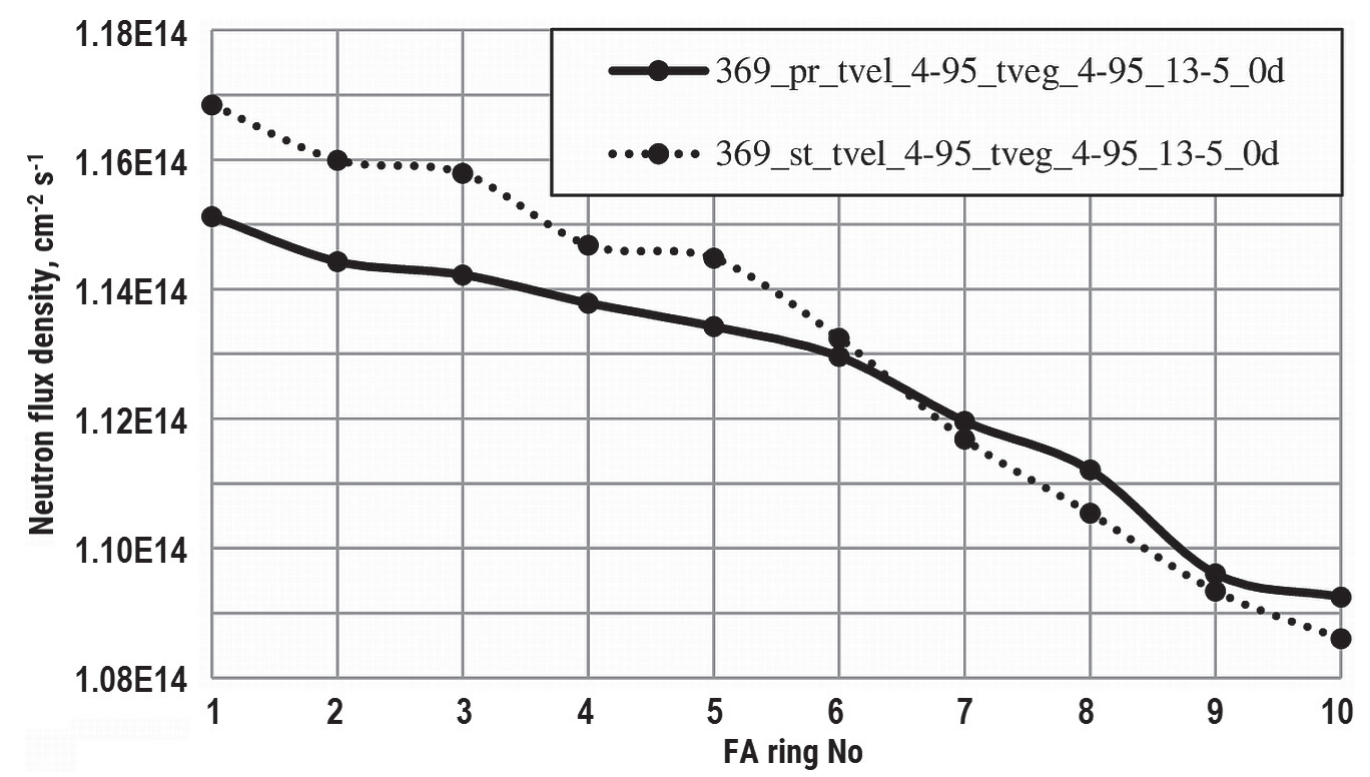

Figure 3. Effect of profiling on the neutron flux density at $t=0$ eff. days.

An increase in enrichment up to $5.2 \%$, as well as gadolinium oxide content of $13.5 \%$, makes the assembly with two rings the most promising one during a microcampaign of less than 500 eff. days. Between 500 and 560 eff. days, the version with profiling of three Gd fuel element rings is the most optimal of the considered ones. With a cycle of more than 560 days, the standard arrangement of gadolinium oxide is more efficient.

An insignificant (relative to higher enrichment when Versions 1 and 2 are considered) increase in the amount of uranium-235 in fuel assemblies of Version 7 due to higher enrichment in fuel elements further does not affect the behavior of $k_{\text {eff }}$ during a campaign with a given amount of gadolinium and arrangement format. A noteworthy detail is that, in Versions 6 and 7, a clear advantage of the assembly with two rings was observed for a long time, but the version with the profiled arrangement of gadolinium oxide in three rings is more uniformly burning.

A further increase in fuel enrichment (in Version 8) leads to the possibility of extending the duration of a single cycle from the standpoint of assessing the efficiency of using a profiled version of fuel assemblies. Moreover, the profiled arrangement of a burnable absorber in two rings is more effective for 550 eff. days in comparison with the arrangement in three rings and for 610 eff. days in comparison with the standard arrangement. The profiled arrangement of Gd fuel elements in three hexagonal rings of fuel assemblies is effective up to 660 eff. days.

An increase in the content of gadolinium oxide in fuel assemblies (Versions 9-11) leads to a shift in the point of intersection of the $k_{\text {eff }}$ of the profiled and standard assemblies, regardless of the placement. Considering the neutronic side of the issue, we see that with a proportional increase in fuel enrichment and gadolinium content in $\mathrm{Gd}$ fuel elements, the fuel cycle for the profiled version can be more than 860 eff. days (enrichment $=5.45 \%, \mathrm{Gd}_{2} \mathrm{O}_{3}$ content $=24 \%$ ).

The second interesting aspect is the change in the distribution of the neutron flux density along the radius of the fuel assembly. For this, the average value of the total neutron flux density was determined in each ring. The calculated results are offered for Version 5 at the initial moment of time (Fig. 3) and at the moment $t=$ 560 eff. days (Fig. 4). The reference points are chosen according to the behavior of $k_{\text {eff }}$ (the points are close to the moment of intersection of the $k_{\mathrm{eff}}$ lines of the standard and profiled assemblies).

For all the versions considered, the gradient of the neutron flux density for the initial moment of time became smaller for the profiled version of the assemblies. However, by the time the efficiency of fuel assembly profiling decreases, the gradient of the original assembly becomes less or similar to the profiled one. This is probably due to the uneven burnup of Gd fuel elements in different rings. Visually, this can be observed in Figs 5, 6 .

The result is indicative when the concentration of Gd155 tends to zero already at 200 eff. days, while for the outer ring, the concentration of both isotopes by the time of 360 eff. days is still significant. Thus, the isotopic imbalance leads to an uneven neutron flux density at the time when the standard assembly is more efficient than the profiled one.

\section{Conclusion}

The results of the work indicate the advantage of using profiling for assemblies with three rings of $\mathrm{Gd}$ fuel elements. At the same time, in the case of uniform distribution of gadolinium in Gd fuel elements, the assemblies with three-ring and two-ring arrangements have identical $k_{\text {eff }}$ at different times. 


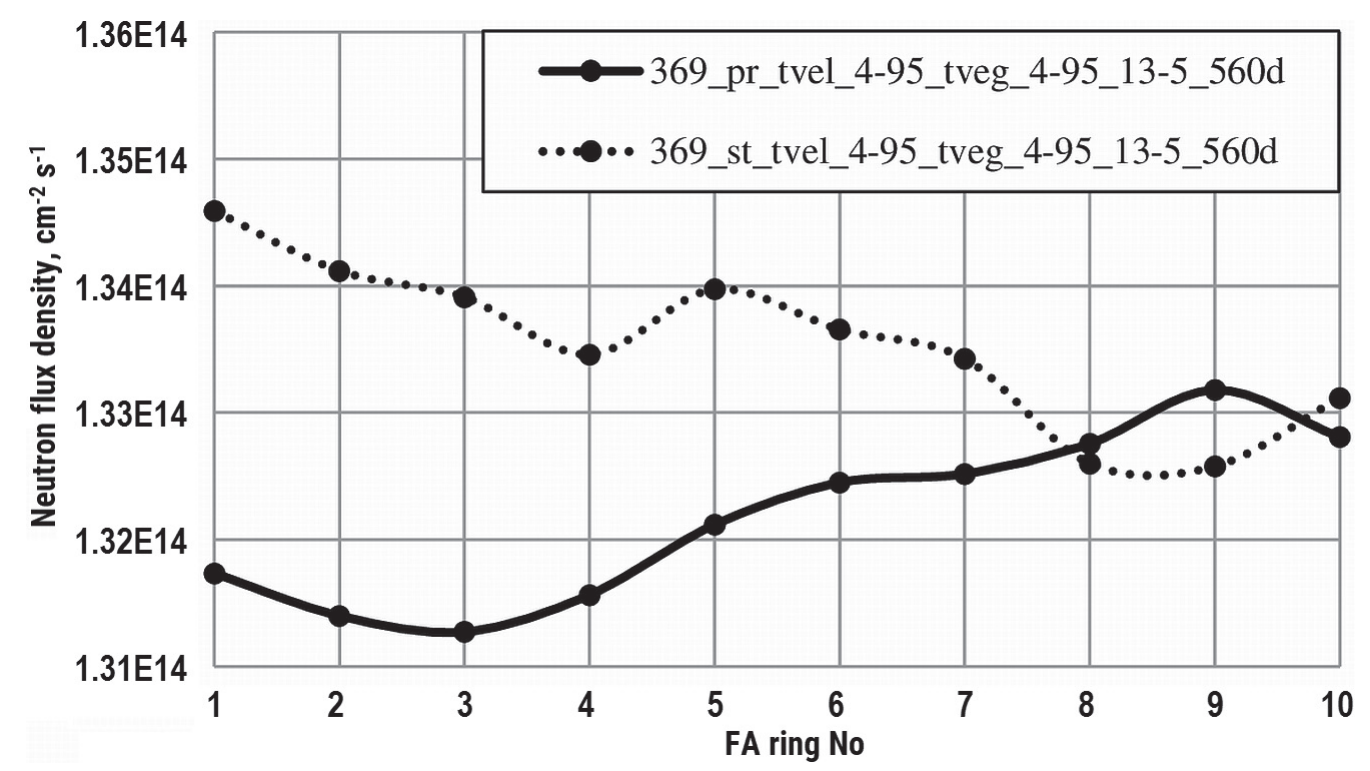

Figure 4. Effect of profiling on the neutron flux density at $t=560$ eff. days.

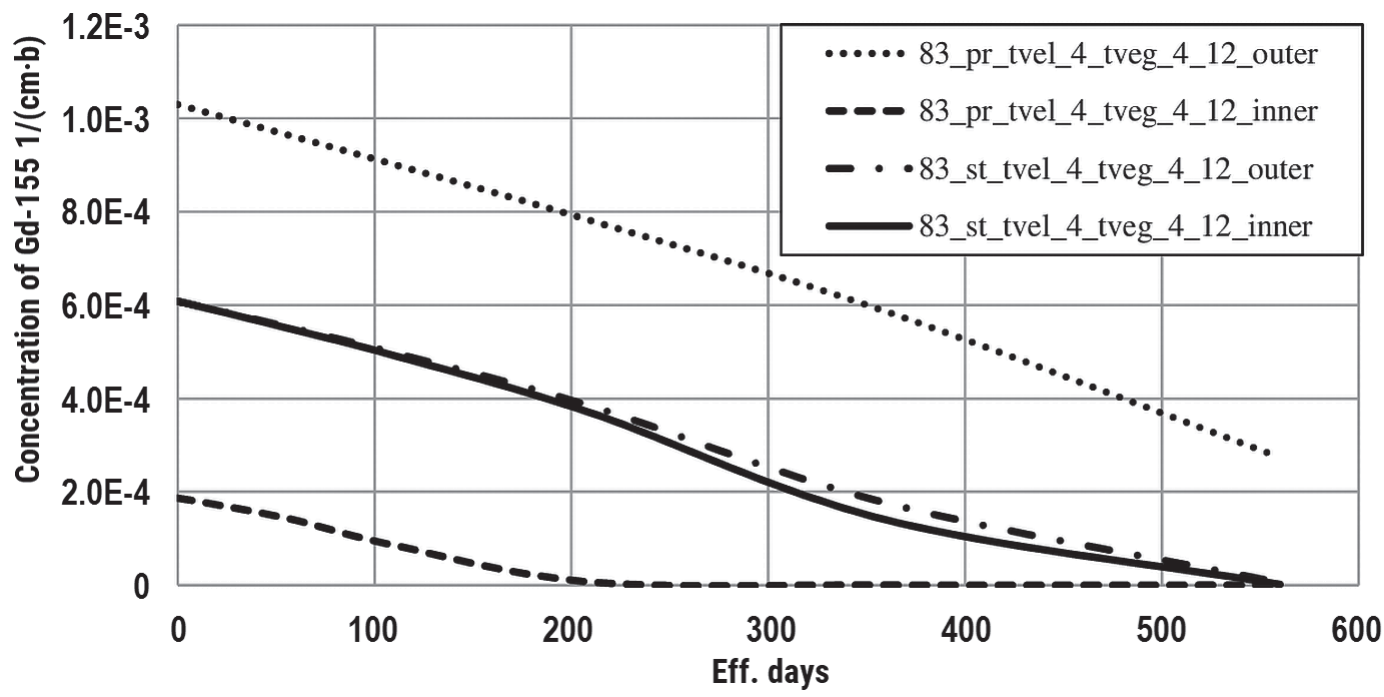

Figure 5. Change in the isotopic composition of Gd-155 for arranging Gd fuel elements in two rings (enrichment $=4 \%, \mathrm{Gd}_{2} \mathrm{O}_{3}$ content in Gd fuel elements $=12 \%$ ).

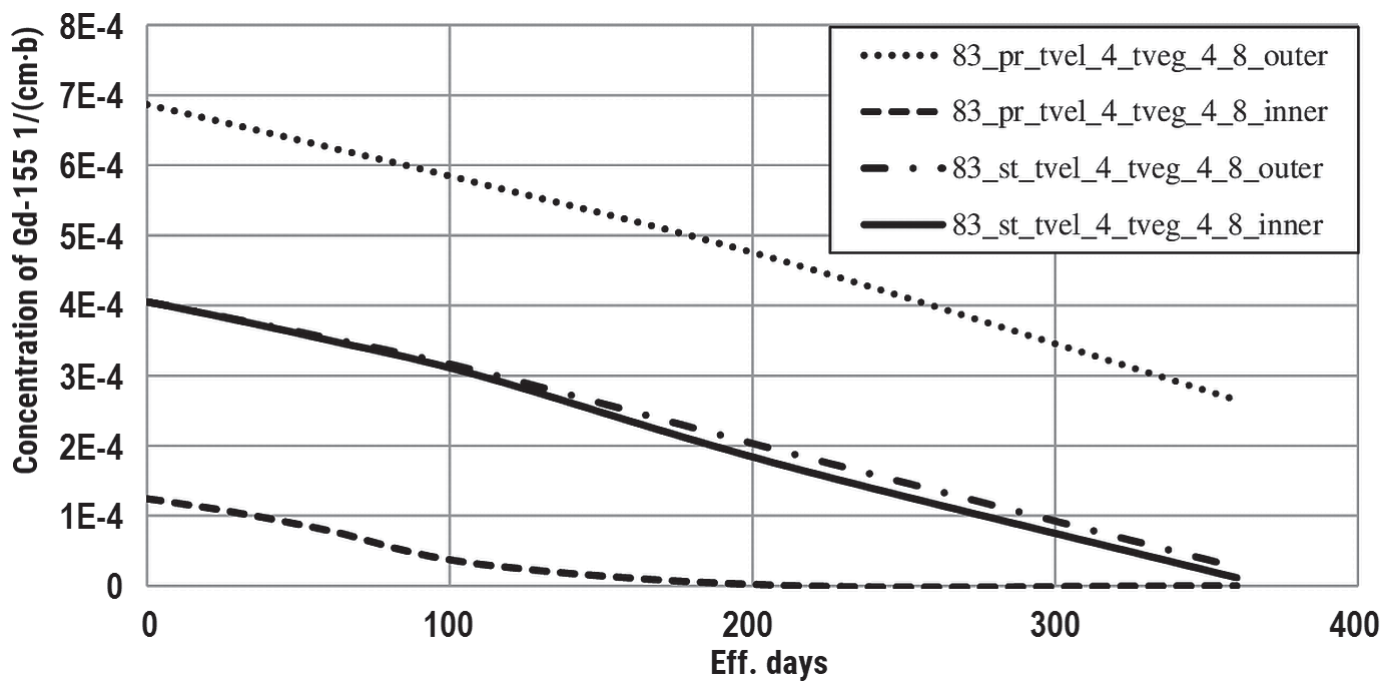

Figure 6. Change in the isotopic composition of Gd-155 for arranging Gd fuel elements in two rings (enrichment $=4 \%, \mathrm{Gd}_{2} \mathrm{O}_{3}$ content in Gd fuel elements $=8 \%$ ). 
An increase in the amount of gadolinium at the moment favorably affects the leveling of $k_{\text {eff }}$ in the process of fuel burnup. As fuel enrichment grows up, the effect of profiling a burnable absorber along fuel assemblies increases.

An increase in the amount of gadolinium reduces the total amount of uranium; therefore, at the same enrichment, a moment may come when a further increase in the $\mathrm{Gd}_{2} \mathrm{O}_{3}$ content will lead to a deterioration in the profiling efficiency. According to the calculation results, such a moment will come when the gadolinium content becomes so high as to exceed the technological threshold for the performance of uranium-gadolinium fuel.

\section{Acknowledgements}

Authors acknowledge support of MEPhI Academic Excellence Project (contract No. 02.a03.21.0005, 27.08.2013).

\section{References}

- Abu Sondos MA, Demin VM, Savander VI (2019a) The Effect of Burnable Absorber ( $\mathrm{Gd}$ and $\mathrm{Eu}$ ) on the Neutron-Physics Characteristic of Fuel Assemblies of VVER-1000 Reactor. IOP Conference Series: Journal of Physics: Conference Series. https://doi. org/10.1088/1742-6596/1189/1/012003

- Abu Sondos MA, Demin VM, Savander VI (2019b) Reduction of Boron Reactivity Reserve Control when using a $\mathrm{Gd}_{2} \mathrm{O}_{3}$-based Burnup Absorber in the VVER-1200 Reactor Fuel. Global Nuclear Safety 3(32): 56-65. https://doi.org/10.26583/gns-2019-03-06 [in Russian]

- Abu Sondos MA, Demin VM, Smirnov AD (2019c) Comparative Analysis of Neutronic Characteristics of Nuclear Fuel Produced by Westinghouse and Fuel Rods for VVER-1000-type Reactors by SERPENT Code. Global Nuclear Safety 2(31): 103-109. https://doi. org/10.26583/gns-2019-02-12 [in Russian]

- Frybortova L (2019) Recommended Strategy and Limitations of Burnable Absorbers used in VVER Fuel Assemblies. Nuclear Science and Technology 30(8): e14. https://doi.org/10.1007/s41365-019-0651-x

- IAEA [International Atomic Energy Agency] (2009) Advanced Fuel Pellet Materials and Fuel Rod Design for Water Cooled Reactors. Proc. of the Technical Committee Meeting on 23-26 Nov. IAEA, Villigen, 241 pp. https://www-pub.iaea.org/MTCD/Publications/ PDF/te_1654_web.pdf [accessed Nov. 06, 2020]

- IAEA [International Atomic Energy Agency] (2011) Status Report for Advanced Nuclear Reactor Designs - Report 108 "VVER1200 (V491) (VVER-1200 (V-491))”. IAEA, Vienna, 32 pp. http:// www.iaea.org/NuclearPower/Downloadable/aris/2013/36.VVER1200(V-491).pdf [accessed Nov. 06, 2020]

- IAEA [International Atomic Energy Agency] (2013) Operation and Licensing of Mixed Cores in Water Cooled Reactors. IAEA, Vienna, 90 pp. [IAEA-TECDOC series, ISSN 1011-4289; no. 1720] https:// www-pub.iaea.org/MTCD/Publications/PDF/TE-1720_web.pdf [accessed Nov. 06, 2020]

- Iwasaki K, Matsui T, Yanai K, Yuda R, Arita Y, Nagasaki T, Yokoyama N, Tokura I, Une K, Harada K (2009) Effect of $\mathrm{Gd}_{2} \mathrm{O}_{3}$
Dispersion on the Thermal Conductivity of $\mathrm{UO}_{2}$. Nuclear Science and Technology 46(7): 673-676. https://doi.org/10.1080/18811248 .2007.9711574

- Khoshahval F, Foroutan SS, Zolfaghari A, Minuchehr H (2016) Evaluation of Burnable Absorber Rods Effect on Neutronic Performance in Fuel Assembly of VVER-1000 Reactor. Annals of Nuclear Engeneering 87: 648-658. https://doi.org/10.1016/j. anucene.2015.10.012

- Kryukov FN, Lyadov GD, Nikitin ON (2005) Investigation of the Fuel State of Thermal Neutron Reactors by Electron-Probe Microanalysis. VANT. Ser.: Nuclear Reactor Physics 5: 94-103. [in Russian]

- Leppänen J, Pusa M, Viitanen T, Valtavirta V, Kaltiaisenaho T (2015) The SERPENT Monte Carlo Code: status, development and applications in 2013. Annals of Nuclear Energy 82: 142-150. https://doi. org/10.1016/j.anucene.2014.08.024

- Saad HM, Refeat R, Aziz M, Mansour H (2019) Effect of axial distribution of gadolinium burnable poison in advanced pressurized water reactor assembly. Nuclear and Radiation Safety Journal 84(4): 46-53. https://doi.org/10.32918/nrs.2019.4(84).06

- SERPENT - MCRPBCC (2020) SERPENT - MCRPBCC [A Continuous-Energy Monte Carlo Reactor Physics Burnup Calculation Code]. http://montecarlo.vtt.fi [accessed Nov. 06, 2020]

- Slivin AA, Anikin MN, Chertkov YuB (2016) Possibility of Alternative Burnup Absorbers in VVER-type Nuclear Reactors. In: Proceedings of the Conf. On Actual Problems of Innovative Development of Nuclear Technologies, March 21-25, 2016. Seversk. Federal State Educational Institution of Higher Professional Education NRNU MEPhI. Seversk Institute of Technology Publ., 51. [in Russian]

- Stefanova S, Chantoin P, Kolev IG (1994) VVER Reactor Fuel Performance. Modelling and Experimental Support. In: Proceedings of the International Seminar held in St. Constantine, Varna, Bulgaria on 7-11 Nov. 1994. Varna, Bulgaria, 260 pp. https://inis.iaea.org/ collection/NCLCollectionStore/_Public/28/031/28031101.pdf [accessed Nov. 06, 2020] 